\title{
MicroRNA-22 may promote apoptosis and inhibit the proliferation of hypertrophic scar fibroblasts by regulating the mitogen-activated protein kinase kinase/extracellular signal-regulated kinase/p21 pathway
}

\author{
SHIHUA DONG and YANFENG SUN
}

Department of Burn and Plastic Surgery, Daqing Oilfield General Hospital, Daqing, Heilongjiang 163001, P.R. China

Received September 22, 2016; Accepted June 5, 2017

DOI: $10.3892 /$ etm.2017.4942

\begin{abstract}
Hypertrophic scarring (HS) is a common skin disorder that occurs during the wound healing process, and the pathogenesis of HS remains unclear. Increasing evidence indicated that specific microRNAs (miRs) may be involved in the onset and progression of HS. In the present study, the association between miR-22 and HS was investigated. Reverse transcription-quantitative polymerase chain reaction (RT-qPCR) was performed to examine the expression of miR-22 in $30 \mathrm{HS}$ and matched normal skin tissues. In addition, human hypertrophic scar fibroblasts (HSFBs) were cultured and transfected with miR-22 mimics, and MTT and Annexin V apoptosis assays were performed to investigate the role of miR-22 in the proliferation and apoptosis of the human HSFBs. Next, RT-qPCR and western blot assays were performed to compare the expression levels of mitogen-activated protein kinase kinase (MEK), extracellular signal-regulated kinase (ERK) and p21 in untransfected and miR-22 mimic-transfected skin fibroblasts. The results identified that miR-22 was significantly downregulated in HS tissues as compared with the normal skin. Furthermore, transfection with miR-22 mimics in human HSFBs led to inhibited cell proliferation, increased apoptosis, as well as to decreased MEK expression and ERK1/2 phosphorylation, and increased expression of p21. In conclusion, the present study was the first to prove that aberrant expression of miR-22 may serve an important role in the pathogenesis of HS by regulating the MEK/ERK/p21 pathway, thus suggesting that miR-22 has the potential to become a therapeutic target for the treatment of HS.
\end{abstract}

Correspondence to: Dr Yanfeng Sun, Department of Burn and Plastic Surgery, Daqing Oilfield General Hospital, 9 Zhongkang Street, Saertu, Daqing, Heilongjiang 163001, P.R. China E-mail: dr_yanfengsun@hotmail.com

Key words: miR-22, p21, mitogen-activated protein kinase kinase, extracellular signal-regulated kinase, hypertrophic scarring

\section{Introduction}

Hypertrophic scarring (HS) is a cutaneous condition characterized by prominent scar tissue above the skin, caused by abnormal expression of disordered collagens in the skin fibroblasts (1). HS usually develops during the wound healing process subsequent to skin trauma or severe burn injury, resulting in physiological and psychological problems for patients (2). Previous studies indicated that the transforming growth factor- $\beta 1$ signaling pathway may be involved in the pathogenesis of wound healing and scar formation by promoting cellular proliferation and the progression of fibrosis (3). However, the underlying molecular mechanism of HS development remains poorly understood. Current treatment of HS mainly depends on scar revision surgery, which is an invasive and painful method; thus, there is an urgent need to identify therapeutic targets and effective medications for the management of HS.

MicroRNAs (miRNAs or miRs) are small non-coding RNAs that negatively regulate the expression of genes through silencing (by complementary binding to the 3'-untranslated region of the mRNA) or direct cleaving of the target mRNAs. Previous studies have demonstrated that miRNAs participate in multiple cellular and molecular activities, including cell proliferation, differentiation and apoptosis, embryo development and tumorigenesis (4). In the field of dermatology, increasing evidence indicated that miRNAs are involved in the mechanism of skin development and the pathogenesis of certain skin disorders $(5,6)$. It has been reported that miR-125b was downregulated in psoriatic lesions, while transient overexpression of miR-125b induced significant decrease in the proliferation of human keratinocytes through directly targeting fibroblast growth factor receptor 2 (7). These results indicated that miR-125b may serve a protective role in psoriasis, while decreased expression of miR-125b in the epidermis may lead to the hyperproliferation of keratinocytes (7). The roles of specific miRNAs in HS or keloid formation have also been investigated in previous studies. For instance, miR-21 was found to be upregulated in HS tissue, while silencing of miR-21 promoted cell proliferation and inhibited apoptosis in hypertrophic scar fibroblasts (HSFBs) in vitro and in vivo $(8,9)$. Furthermore, overexpression of 
miR-200b inhibited the proliferation and promoted the apoptosis of human HSFBs in vitro (10). Gras et al (11) observed increased expression of miR-145 in HS tissues, and also proved that silencing of miR-145 significantly reduced myofibroblast activity, suggesting that miR-145 has the potential to become a therapeutic target for treating HS. However, thus far, the roles of different miRNAs in the pathogenesis of HS require further investigation.

miR-22 has been long considered as a novel tumor suppressor, and the protective roles of miR-22 in skin diseases, such as cutaneous T-cell lymphoma (12) and melanoma (13), have previously been discussed. To the best of our knowledge, the association between miR-22 and HS has not yet been investigated. In the present study, we demonstrated the effects of miR-22 on the apoptosis and proliferation of hypertrophic scar fibroblasts by regulating the mitogen-activated protein kinase kinase/extracellular signal-regulated kinase/p21 pathway.

\section{Materials and methods}

Tissue samples. A total of 30 HS tissue samples and a matched number of adjacent normal skin tissues were obtained from patients during plastic surgery performed at the Daqing Oilfield General Hospital (Daqing, China). The diagnosis of HS was confirmed using routine pathological approaches. All experiments were conducted subsequent to obtaining approval of the Ethics Committee of Daqing Oilfield General Hospital, and all patients were required to sign the informed consent forms for inclusion into the current study.

Cell culture. Human hypertrophic scar fibroblasts (HSFBs) (Shanghai Bioleaf Biotech Co., Ltd., Shanghai, China) were cultured in Dulbecco's modified Eagle's medium (Thermo Fisher Scientific, Inc., Waltham, MA, USA) supplemented with $10 \%$ fetal bovine serum (Thermo Fisher Scientific, Inc.) and $1 \%(\mathrm{w} / \mathrm{v})$ penicillin/streptomycin (Sigma-Aldrich; Merck, Darmstadt, Germany) in a $5 \% \mathrm{CO}_{2}$ humidified incubator at $37^{\circ} \mathrm{C}$.

Cell transfection. Human HSFBs were transfected with $50 \mathrm{nmol}$ miR-22 mimics (GenePharma Co.,Ltd., Shanghai, China) using Lipofectamine 3000 (Invitrogen; Thermo Fisher Scientific, Inc.) according to the manufacturer's protocol. The sequences of the antisense oligonucleotides were used: miR-22 mimics, forward 5'-CTCAACTGGTGTCGTGGAGTCGG-3' and reverse 5'-CAATTCAGTTGAGACAGTTCT-3'; and miRNA control, forward 5'-UUCUCCGAACGUGUCACGUTT-3' and forward 5'-ACGUGACACGUUCGGAGAATT-3' for the miRNA mimics control. Cells were harvested at $72 \mathrm{~h}$ after transfection and used in subsequent analyses.

Reverse transcription-quantitative polymerase chain reaction $(R T-q P C R)$. Total RNA was extracted using TRIzol reagent, and RNA purification and the concentration were measured using a Nanodrop 2000 instrument (both from Thermo Fisher Scientific, Inc.). RT was performed using $2 \mu \mathrm{l}$ RNA with PrimeScript $^{\mathrm{TM}}$ RT reagent kit (Takara Biotechnology Co., Ltd., Dalian, China). Subsequently, qPCR was conducted using SYBR $^{\circledR}$ Premix Ex Taq ${ }^{\mathrm{TM}}$ kit (Takara Biotechnology Co., Ltd.) on an ABI 7300 Real-Time PCR system (Applied Biosystems;
Thermo Fisher Scientific, Inc.) according to the manufacturer's protocol. The thermal cycling profiles were as follow: $95^{\circ} \mathrm{C}$ for $30 \mathrm{sec}$, followed by 40 cycles of $95^{\circ} \mathrm{C}$ for $5 \mathrm{sec}$ and $60^{\circ} \mathrm{C}$ for $30 \mathrm{sec}$. Primer sequences were synthesized by GenScript Co., Ltd. (Nanjing, China) and were as follows: p21 forward, 5'-ATGTCAGAACCGGCTGGGGA-3', and reverse, 5'-TTAG GGCTTCCTCTTGGAGA-3'; MEK forward, 5'-GACGACCA GTGGGGAGAGTA-3', and reverse, 5'-GTCATTGAGCCGA CCTAA-3'; ERK1 forward, 5'-TCCTTTGGATCTGGTCCTG-3', and reverse, 5'-CCCCAGCAAGTGAGAGAAG-3'; ERK2 forward, 5'-AAGAGGTTGTTCCAAATGC-3', and reverse, 5'-AGAGGCACCATTCACTGAC-3'; and GAPDH forward, 5'-AAGTATGACAACAGCCTCAAGA-3', and reverse, 5'-CACCACCTTCTTGATGTCATCA-3'. The relative expression of p21, MEK, ERK1 and ERK2 genes in each sample was normalized to the level of GAPDH using the $2^{-\Delta \Delta \mathrm{Cq}}$ method (14).

The expression of miR-22 was examined using the Hairpin-it miRNAs qPCR Quantitation kit (GenePharma Co., Ltd.) according to the manufacturer's protocol. U6 (RNU6B; GenePharma Co., Ltd.) was used for normalization of the miR-22 expression.

Western blot analysis. Proteins were extracted from the harvested cells using radioimmunoprecipitation assay (Beyotime Institute of Biotechnology, Shanghai, China), and the concentration of the protein was determined using Pierce BCA Protein Assay kit (Thermo Fisher Scientific, Inc.). Next, the proteins were separated using $12.5 \%$ SDS-PAGE, and transferred onto polyvinylidene fluoride membranes, followed by blocking using 5\% non-fat milk in Tris-buffered saline at room temperature for $1 \mathrm{~h}$. Membranes were then incubated at $4^{\circ} \mathrm{C}$ overnight with the following primary antibodies: Anti-MEK (cat. no. ab96379; 1:1,000), anti-p-ERK1/2 (cat. no. ab17942; 1:1,000), anti-p21 (cat. no. ab109520; 1:1,000) and $\beta$-actin (cat. no. ab8226; 1:1,000; all purchased from Abcam, Cambridge, MA, USA). Subsequently, the membranes were washed with Tris-buffered saline with Tween-20 buffer and incubated with secondary antibody (anti-rabbit-HRP; cat. no. ab6721; 1:2,000; Abcam) at room temperature for $1 \mathrm{~h}$. Luminescence was revealed by incubation with Novex ECL Chemiluminescent Substrate Reagent kit (Thermo Fisher Scientific, Inc.), and the signals were detected using ChemiDoc ${ }^{\mathrm{TM}} \mathrm{XRS}+$ imaging system (Bio-Rad Laboratories, Inc., Hercules, CA, USA).

Cell apoptosis assay by flow cytometry. Cells was stained with the Annexin V/propidium iodide apoptosis detection kit (BD Biosciences, Franklin Lakes, NJ, USA) at $72 \mathrm{~h}$ after transfection, following the manufacturer's instructions. The apoptosis rate of cells was then analyzed using BD FACSVerse flow cytometer (BD Biosciences).

Cell proliferation assay. MTT assay was performed at $72 \mathrm{~h}$ after transfection to determine the cell viability using a MTT proliferation assay kit (Sigma-Aldrich; Merck) according to the manufacturer's instructions.

Statistical analysis. Statistical analysis was conducted using SPSS software version 21.0 (IBM Corp., Armonk, NY, USA). Data are presented as the means \pm standard deviation, and differences were examined using Student's t-test. $\mathrm{P}<0.05$ was 


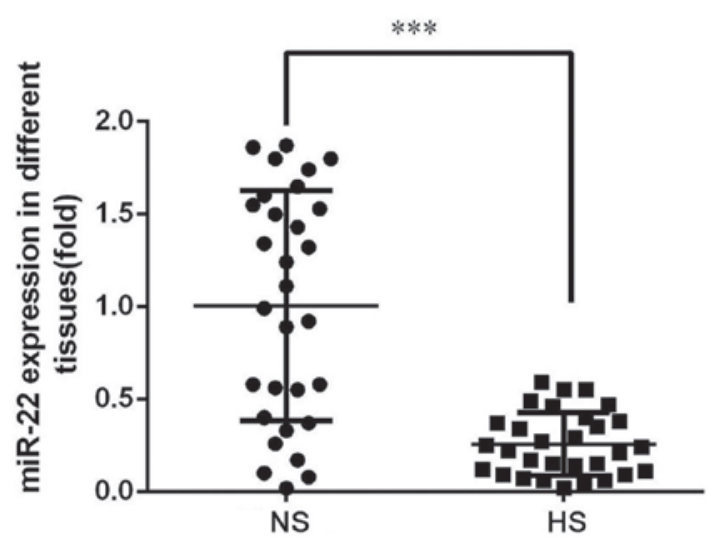

Figure 1. Relative expression of miR-22 in NS and HS tissue samples. Data are presented as the mean \pm standard deviation. ${ }^{* * *} \mathrm{P}<0.001$. miR, microRNA; NS, normal skin; HS, hypertrophic scarring.

considered to demonstrate differences that were statistically significant.

\section{Results}

Decreased expression of miR-22 in HS tissues. In order to investigate the role of miR-22 in HS, the present study initially compared the expression of miR-22 in human HS tissue samples and normal skin tissues using RT-qPCR. As shown in Fig. 1, the expression of miR-22 was significantly decreased in the majority of the HS tissue samples as compared with the levels in normal skin $(\mathrm{P}<0.001)$.

Overexpression of miR-22 inhibits proliferation and increases apoptosis of human HSFBs. Currently, it is generally accepted that HS is caused by excessive proliferation of HSFBs (9). Thus, to further explore the function of miR-22 in HS, HSFBs were transfected with miR-22 mimics, and cell proliferation and apoptosis analyses were then conducted. As shown in Fig. 2, compared with the normal cells (NC) and miR-22 empty vector transfected cells (blank group, BL), the transient overexpression of miR-22 induced by mimic transfection significantly inhibited the cell proliferation $(\mathrm{P}<0.01$; Fig. $2 \mathrm{~A})$ and promoted the cell apoptosis ( $\mathrm{P}<0.001$; Fig. $2 \mathrm{~B})$.

miR-22 may regulate the expression levels of MEK, $p-E R K$ and $p 21$ in HSFBs. The possible mechanism underlying the effect of miR-22 on the proliferation and apoptosis of HSFBs was also explored. The expression levels of certain key molecules that are involved in the proliferation and apoptosis signaling pathway were examined using RT-qPCR and western blot assays. As observed in Fig. 3, the RT-qPCR results indicated that the mRNA expression of $M E K$ was significantly increased (Fig. 3A; $\mathrm{P}<0.001$ ) and the mRNA expression of p21 was markedly reduced (Fig. 3D; $\mathrm{P}<0.001)$ in the miR-22 group compared with the NC and BL groups. By contrast, the expression levels of ERK1 (Fig. 3B) and ERK2 (Fig. 3C) presented no significant differences at the mRNA level among the three groups. Furthermore, compared with the NC and BL groups, transient overexpression of miR-22 in HSFBs induced a significant decrease in the expression levels of MEK and phosphorylated ERK1/2 (p-ERK1/2), as well as a
A

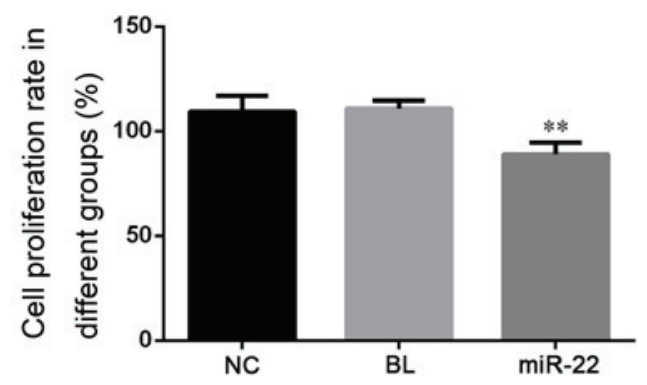

B
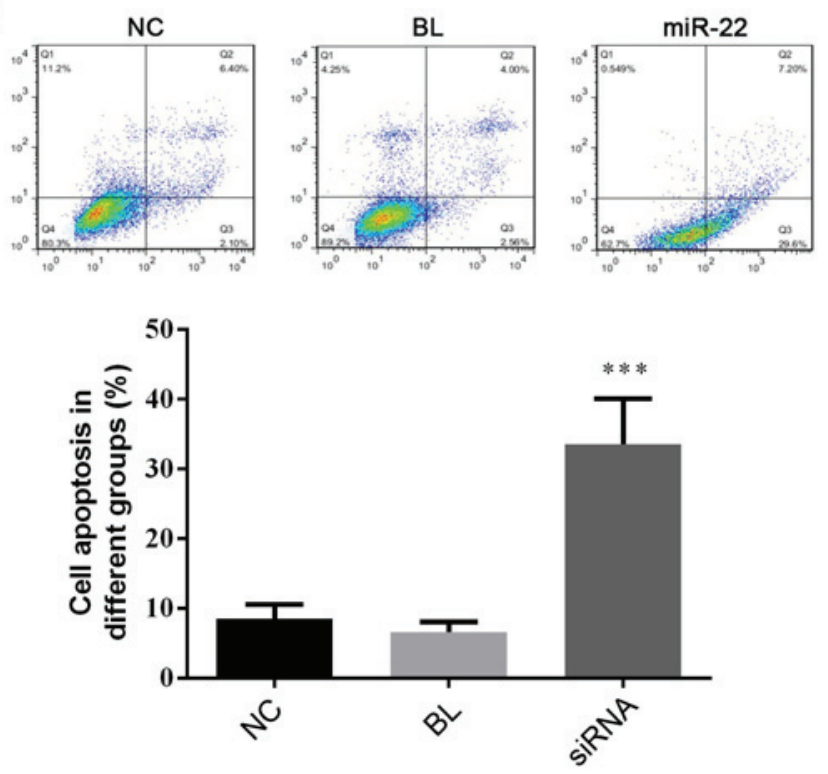

Figure 2. Transfection of human HSFBs with miR-22 mimics inhibits the proliferation and increases apoptosis of cells. (A) Cell proliferation rate and (B) apoptosis of HSFBs in the NC, BL and miR-22 groups. Data are presented as the mean \pm standard deviation. ${ }^{* *} \mathrm{P}<0.01$ and ${ }^{* * *} \mathrm{P}<0.001$ vs. NC and BL groups. miR, microRNA; HSFBs, hypertrophic scar fibroblasts; NC, normal cells; BL, blank group; miR-22, transfected with miR-22 mimics.

significant increase in the expression of $\mathrm{p} 21$ at the protein level (all $\mathrm{P}<0.01$; Fig. 4). These results indicated that miR-22 may affect the proliferation and apoptosis of HSFBs by regulating the MEK/ERK/p21 signaling pathway.

\section{Discussion}

The roles of miR-22 in skin development and skin diseases have been discussed in various previous studies. For instance, Yuan et al (15) reported that miR-22 regulates the proliferation and differentiation of keratinocyte progenitor cells and participates in the mechanism of hair follicle regression. Sibbesen et al (12) demonstrated that Janus kinase 3, signal transducer and activator of transcription 3 (STAT3) and STAT5 inhibited the expression of miR-22 in cutaneous T-cell lymphoma. In addition, Sand et al (16) discovered that miR-22 was significantly upregulated in melanoma, suggesting that miR-22 may be involved in the pathogenesis of the disease. However, to the best of our knowledge, the expression and functions of miR-22 in HS have yet to be discussed. In the present study, it was initially proven that miR-22 was downregulated in human HS tissues as compared with normal skin, and notably, transient overexpression of miR-22 in human HSFBs induced a significant decrease in cell proliferation and 
A

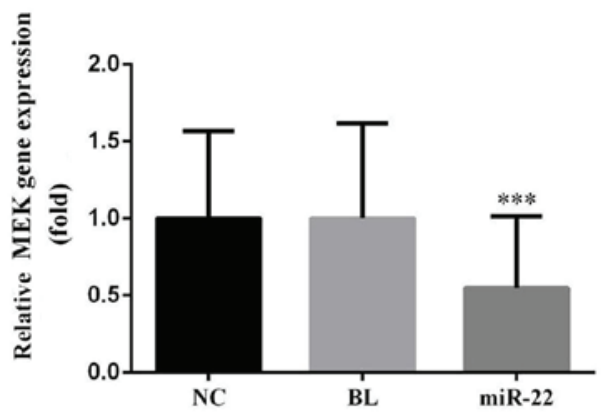

B

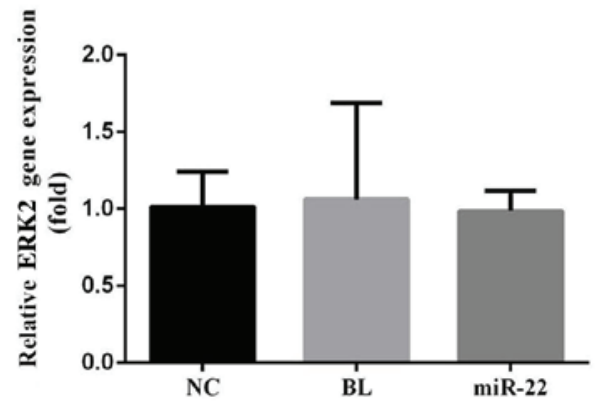

C

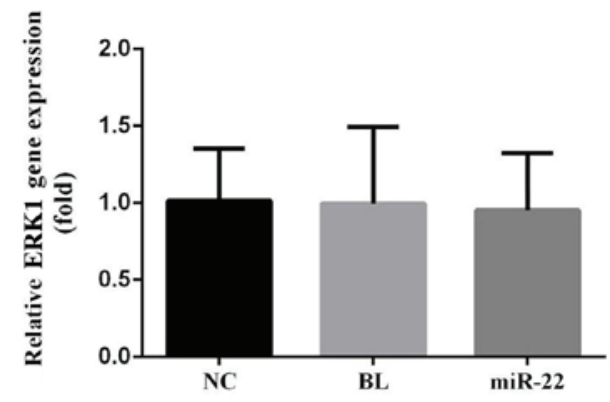

D

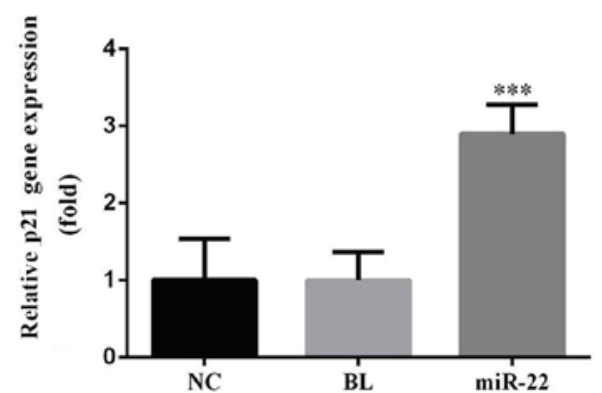

Figure 3. Relative gene expression levels of (A) $M E K$, (B) ERK2, (C) ERK1 and (D) p21 in hypertrophic scar fibroblasts in the NC, BL and miR-22 groups. ${ }^{* * *} \mathrm{P}<0.001$ vs. NC and BL groups. MEK, mitogen-activated protein kinase; ERK, extracellular signal-regulated kinase; NC, normal cells; BL, blank group, transfected with miR-22 empty vector; miR-22, transfected with miR-22 mimics.

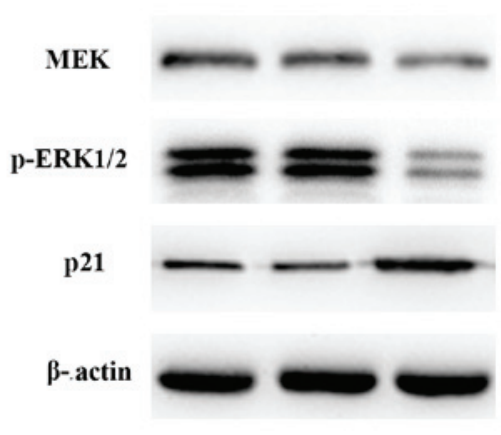

p-ERK1/2

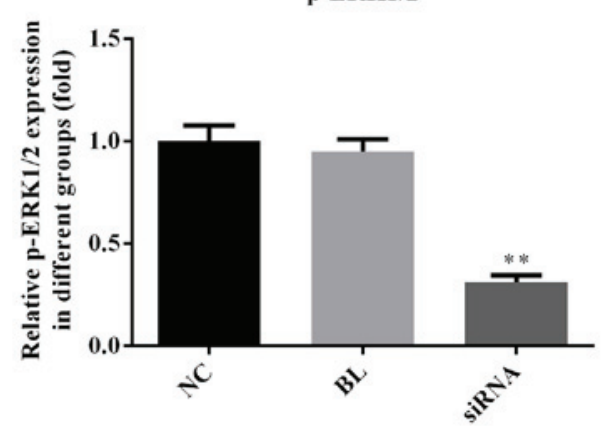

MEK

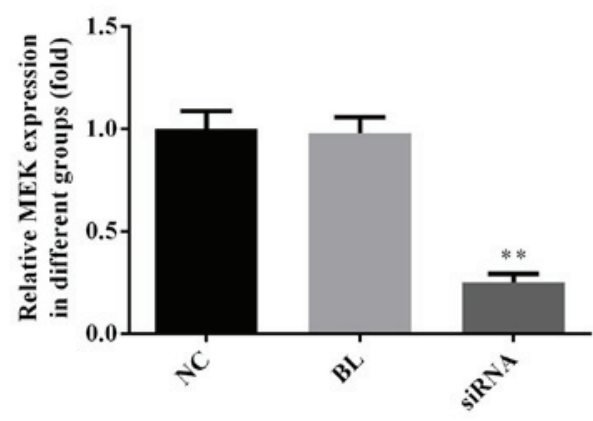

p21

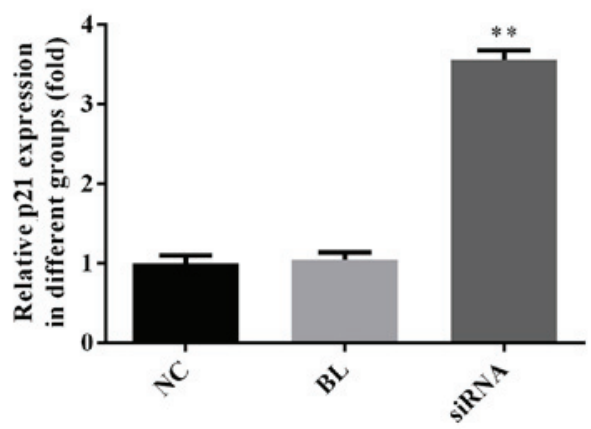

Figure 4. Western blot analysis demonstrating the relative levels of MEK, p-ERK1/2 and p21 proteins in hypertrophic scar fibroblasts. ** P $<0.01$ vs. NC and BL groups. MEK, mitogen-activated protein kinase; p-ERK1/2, phosphorylated extracellular signal-regulated kinase 1/2; NC, normal cells; BL, blank group; miR-22, transfected with miR-22 mimics.

increase in cell apoptosis. Since HS is commonly considered as an epidermal hyperplasia resulting from excessive growth of HSFBs, the present study data provided novel evidence that miR-22 was aberrantly expressed in human HS, as well as that 
decreased expression of miR-22 in HS may lead to increased proliferation and decreased apoptosis of HSFBs.

Previous studies have demonstrated that $\mathrm{p} 21^{\mathrm{CIP} 1 / \mathrm{WAF}}$, a cyclin-dependent kinase inhibitor that negatively regulates the cell cycle, served important roles in the mechanism of cell growth and senescence $(17,18)$. Increased expression of $\mathrm{p} 21^{\mathrm{CIP} 1}$ can lead to the arrest of the cell cycle at G0/G1 phase (17), while $\mathrm{p} 21^{\mathrm{CIP} 1}$ has also been identified as a key regulator of cellular senescence through activating the MEK/ERK pathway in different cells $(19,20)$. The role of p21 and MEK/ERK signaling in HS has been discussed in previous studies. Chang et al (21) demonstrated that 5-aminolevulinic acid-based anti-HS photodynamic therapy induced increased expression of p53/p21 in HSFBs, which may further active the downstream molecules and promoted cell apoptosis. Furthermore, Li et al (22) observed that kynurenine treatment increased the expression of MEK and the phosphorylation of ERK1/2 in human dermal fibroblasts. He et al (23) also demonstrated the role of ERK signaling in loureirin B-induced inhibition of HS formation. To the best of our knowledge, the association between miRNAs and MEK/ERK/p21 signaling in HS has not been discussed thus far. In the current study, it was first reported that transient overexpression of miR-22 in HSFBs induced significant decrease in the expression of MEK and the phosphorylation of ERK1/2, as well as an increase in the expression of $\mathrm{p} 21$, suggesting that miR-22 may affect the proliferation and apoptosis of HSFBs through regulating the $\mathrm{MEK} / \mathrm{ERK} / \mathrm{p} 21$ signaling pathway in HS.

In conclusion, the present study observed a decreased expression of miR-22 in HS, while transient overexpression of miR-22 inhibited the proliferation and promoted the apoptosis of HSFBs. Furthermore, transfection of HSFBs with miR-22 mimics significantly affect the expression levels of MEK, p-ERK1/2 and p21 in. Taken together, the presented data demonstrated for the first time that miR-22 may serve a protective role in human HS through regulating the MEK/ERK/p21 signaling pathway.

\section{References}

1. Gauglitz GG, Korting HC, Pavicic T, Ruzicka T and Jeschke MG: Hypertrophic scarring and keloids: Pathomechanisms and current and emerging treatment strategies. Mol Med 17: 113-125, 2011.

2. Brewin MP and Lister TS: Prevention or treatment of hypertrophic burn scarring: A review of when and how to treat with the pulsed dye laser. Burns 40: 797-804, 2014.

3. Abdou AG, Maraee AH, Al-Bara AM and Diab WM: Immunohistochemical expression of TGF- $\beta 1$ in keloids and hypertrophic scars. Am J Dermatopathol 33: 84-91, 2011.

4. Sayed D and Abdellatif M: MicroRNAs in development and disease. Physiol Rev 91: 827-887, 2011.

5. Fiedler J, Grönniger E, Pfanne A, Brönneke S, Schmidt K, Falk CS, Wenck H, Terstegen L, Thum $\mathrm{T}$ and Winnefeld M: Identification of miR-126 as a new regulator of skin aging. Exp Dermatol 26: 284-286, 2017.
6. Zhang L, Ge Y and Fuchs E: miR-125b can enhance skin tumor initiation and promote malignant progression by repressing differentiation and prolonging cell survival. Genes Dev 28: 2532-2546, 2014

7. Xu N, Brodin P, Wei T, Meisgen F, Eidsmo L, Nagy N, Kemeny L, Ståhle M, Sonkoly E and Pivarcsi A: MiR-125b, a microRNA downregulated in psoriasis, modulates keratinocyte proliferation by targeting FGFR2. J Invest Dermatol 131: 1521-1529, 2011.

8. Li G, Zhou R, Zhang Q, Jiang B, Wu Q and Wang C: Fibroproliferative effect of microRNA-21 in hypertrophic scar derived fibroblasts. Exp Cell Res 345: 93-99, 2016.

9. Zhu HY, Li C, Bai WD, Su LL, Liu JQ, Li Y, Shi JH, Cai WX, Bai XZ, Jia YH, et al: MicroRNA-21 regulates hTERT via PTEN in hypertrophic scar fibroblasts. PLoS One 9: e97114, 2014.

10. Li P, He QY and Luo CQ: Overexpression of miR-200b inhibits the cell proliferation and promotes apoptosis of human hypertrophic scar fibroblasts in vitro. J Dermatol 41: 903-911, 2014.

11. Gras C, Ratuszny D, Hadamitzky C, Zhang H, Blasczyk R and Figueiredo C: miR-145 contributes to hypertrophic scarring of the skin by inducing myofibroblast activity. Mol Med 21: 296-304, 2015.

12. Sibbesen NA, Kopp KL, Litvinov IV, Jønson L, Willerslev-Olsen A, Fredholm S, Petersen DL, Nastasi C, Krejsgaard T, Lindahl LM, et al: Jak3, STAT3, and STAT5 inhibit expression of miR-22, a novel tumor suppressor microRNA, in cutaneous T-Cell lymphoma. Oncotarget 6: 20555-20569, 2015.

13. Luan W, Li L, Shi Y, Bu X, Xia Y, Wang J, Djangmah HS, Liu X, You Y and Xu B: Long non-coding RNA MALAT1 acts as a competing endogenous RNA to promote malignant melanoma growth and metastasis by sponging miR-22. Oncotarget 7 : 63901-63912, 2016.

14. Livak KJ and Schmittgen TD: Analysis of relative gene expression data using real-time quantitative PCR and the 2(-Delta Delta C(T)) method. Methods 25: 402-408, 2001.

15. Yuan S, Li F, Meng Q, Zhao Y, Chen L, Zhang H, Xue L, Zhang $\mathrm{X}$, Lengner $\mathrm{C}$ and $\mathrm{Yu} \mathrm{Z}$ : Post-transcriptional regulation of keratinocyte progenitor cell expansion, differentiation and hair follicle regression by miR-22. PLoS Fenet 11: e1005253, 2015.

16. Sand M, Skrygan M, Sand D, Georgas D, Gambichler T, Hahn SA, Altmeyer P and Bechara FG: Comparative microarray analysis of microRNA expression profiles in primary cutaneous malignant melanoma, cutaneous malignant melanoma metastases, and benign melanocytic nevi. Cell Tissue Res 351: 85-98, 2013.

17. Bunz F, Dutriaux A, Lengauer C, Waldman T, Zhou S, Brown JP, Sedivy JM, Kinzler KW and Vogelstein B: Requirement for p53 and p21 to sustain G2 arrest after DNA damage. Science 282: 1497-1501, 1998.

18. Warfel NA and El-Deiry WS: p21WAF1 and tumourigenesis: 20 years after. Curr Opin Oncol 25: 52-58, 2013.

19. Thaler S, Hahnel PS, Schad A, Dammann R and Schuler M: RASSF1A mediates p21Cip1/Waf1-dependent cell cycle arrest and senescence through modulation of the Raf-MEK-ERK pathway and inhibition of Akt. Cancer Res 69: 1748-1757, 2009.

20. Zhu J, Woods D, McMahon M and Bishop JM: Senescence of human fibroblasts induced by oncogenic Raf. Genes Dev 12: 2997-3007, 1998.

21. Chang M, Ma X, Ouyang T, Lin J, Liu J, Xiao Y, Chen H, Yu J, Huang $\mathrm{Y}$ and $\mathrm{Xu} \mathrm{M}$ : Potential molecular mechanisms involved in 5-Aminolevulinic Acid-based photodynamic therapy against human hypertrophic scars. Plast Reconstr Surg 136: 715-727, 2015.

22. Li Y, Kilani RT, Rahmani-Neishaboor E, Jalili RB and Ghahary A: Kynurenine increases matrix metalloproteinase-1 and -3 expression in cultured dermal fibroblasts and improves scarring in vivo. J Invest Dermatol 134: 643-650, 2014.

23. He T, Bai X, Yang L, Fan L, Li Y, Su L, Gao J, Han S and Hu D: Loureirin B inhibits hypertrophic scar formation via inhibition of the TGF- $\beta 1$-ERK/JNK pathway. Cell Physiol Biochem 37: 666-676, 2015. 